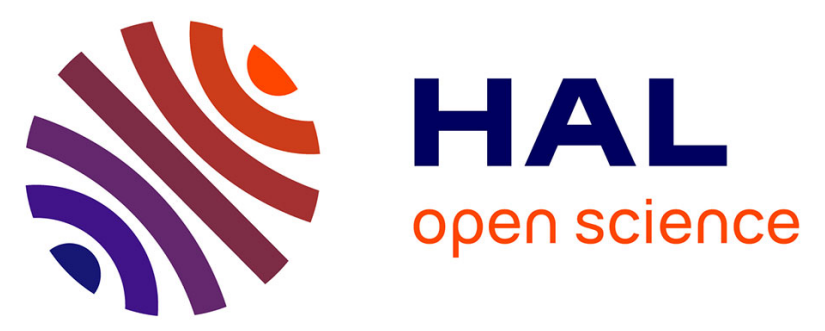

\title{
Molecular dynamics simulations reveal statistics and microscopic mechanisms of water permeation in membrane-embedded artificial water channel nanoconstructs
}

Arthur Hardiagon, Samuel Murail, Li-Bo Huang, Arie van Der Lee, Fabio Sterpone, Mihail Barboiu, Marc Baaden

\section{To cite this version:}

Arthur Hardiagon, Samuel Murail, Li-Bo Huang, Arie van Der Lee, Fabio Sterpone, et al.. Molecular dynamics simulations reveal statistics and microscopic mechanisms of water permeation in membraneembedded artificial water channel nanoconstructs. Journal of Chemical Physics, 2021, 154 (18), pp.184102. 10.1063/5.0044360 . hal-03410227

\section{HAL Id: hal-03410227 \\ https://hal.science/hal-03410227}

Submitted on 9 Nov 2021

HAL is a multi-disciplinary open access archive for the deposit and dissemination of scientific research documents, whether they are published or not. The documents may come from teaching and research institutions in France or abroad, or from public or private research centers.
L'archive ouverte pluridisciplinaire HAL, est destinée au dépôt et à la diffusion de documents scientifiques de niveau recherche, publiés ou non, émanant des établissements d'enseignement et de recherche français ou étrangers, des laboratoires publics ou privés. 


\title{
Molecular dynamics simulations reveal statistics and microscopic mechanisms of water permeation in membrane-embedded artificial water channel nanoconstructs.
}

\author{
Arthur Hardiagon, ${ }^{1}$ Samuel Murail, ${ }^{2}$ Li-Bo Huang, ${ }^{3}$ Arie van der Lee, ${ }^{3}$ Fabio Sterpone,,${ }^{1,4,5}$ Mihail Barboiu, ${ }^{3,5}$ \\ and Marc Baaden ${ }^{1,4,5}$ \\ 1) CNRS, Université de Paris, UPR 9080, Laboratoire de Biochimie Théorique, 13 rue Pierre et Marie Curie, F-75005, Paris, \\ France. \\ ${ }^{2)}$ Université de Paris, CNRS UMR 8251, INSERM ERL U1133, Paris, France \\ ${ }^{3)}$ Institut Européen des Membranes, Adaptive Supramolecular Nanosystems Group, University of Montpellier, ENSCM, CNRS, \\ Place Eugène Bataillon, CC 047, F-34095, Montpellier, France. Email: mihail-dumitru.barboiu@umontpellier.fr \\ ${ }^{4)}$ Institut de Biologie Physico-Chimique-Fondation Edmond de Rotschild, PSL Research University, Paris, \\ France. \\ 5) co-corresponding authors
}

(Dated: 24 March 2021)

Understanding water transport mechanisms at the nanoscale level remains a challenge for theoretical chemical physics. Major advances in chemical synthesis have allowed us to discover new artificial water channels, rivaling with or even surpassing water conductance and selectivity of natural protein channels. In order to interpret experimental features and understand microscopic determinants for performance improvements, numerical approaches based on all-atom molecular dynamics simulations and enhanced sampling methods have been proposed. In this study, we quantify the influence of microscopic observables such as channel radius and hydrogen bond connectivity, and of meso-scale features such as the size of self-assembly blocks, on the permeation rate of a self-assembled nanocrystal-like artificial water channel. Although the absolute permeation rate extrapolated from these simulations is overestimated by one order of magnitude compared to the experimental measurement, the detailed analysis of several observed conductive patterns in large assemblies opens new pathways to scalable membranes with enhanced water conductance for future design.

Artificial Water Channels, nanopores, water dynamics, hydrogen bonds, pore radius.

\section{INTRODUCTION}

Artificial water channels AWCs are synthetic conduits consisting of unimolecular or self-assembled architectures, presenting a central hydrophilic or hydrophobic pore and an outer hydrophobic exterior matching the lipid bilayer or polymeric membrane environments where they are inserted. These constructs ensure the selective water translocation by rejecting ions or other molecules. The development of AWCs has been extensively described during the last decade, toward the highly selective translocation of water-wires / clusters under confined conditions. Most studies on AWCs are devoted to their synthesis, the experimental and theoretical modeling studies on their self-assembly, as well as their transport performances in lipid bilayer membranes ${ }^{1-7}$. Since the structural features of AWCs can be tuned to control specific water recognition, self-assembly and translocation in a biomimetic fashion, most of these endeavors are oriented toward the development of highly selective membranes for water desalination. The single channel permeability is 2 to 3 orders of magnitude lower, similar or even better than for the natural biological reference Aquaporins. However, most AWCs have an important drawback related to their low ion selectivity ${ }^{8}$. The next important step would be mostly related to scale-up issues, by maintaining the single-channel activity observed at the molecular level with single channels in lipid bilayer membranes, toward the fabrication of biomimetic industrial macroscopic $\left(<m^{2}\right)$ membranes. The major challenge of these works relates to the strategy employed to obtain functional high-density distributions of AWCs in a polymeric matrix.

Molecular dynamics (MD) is a powerful tool for understanding phenomena at the nano-scales. Very subtle changes in geometry or chemical structure can produce drastic increase of the water flow in a nanopore, even up to several orders of magnitude ${ }^{9}$. Studying self-assembly in a lipid environment is a challenging task since a variety of structural and dynamical transitions may arise at different characteristic timescales. Experiments measure water or ion flows under out-of-equilibrium conditions at comparatively large timescales (seconds) while most all-atoms molecular dynamics simulations access equilibrium states at much shorter timescales (several microseconds). Therefore, understanding the water transport mechanisms at the atom-level scale through molecular dynamics simulations plays a double role to assist the future design of new chemical materials for a specific target property and to test the current ability of classical models to reproduce water transport in the steady state.

In the present paper, we extend the study of a previously reported artificial self-assembled water channel ${ }^{5,10,11}$. Qualitative features of hydrogen bond network structure, life time, relaxation times and rotational dynamics of water were already described in detail. Here, we now quantitatively address the characterisation of important additional microscopic aspects of distinct water transport regimes under equilibrium conditions. Namely, we studied the structural and dynamic behavior 
of water-wires in variable crystalline patches of the highly permeable artificial water channel, S-octan-2-yl-ureidoimidazole $\mathrm{S}-\mathrm{HC} 8^{10}$.

The main achievement of this study is to clarify the influence of the size and geometry of these structures on the permeation rate. The findings improve our understanding of the ability of self-assembly to form conductive pathways with correlated changes in the pore size (around $2.8 \AA$ ) and the number of hydrogen bonds that water can form in hydrophilic channels. In order to explore the variability of such observables across simulations, we use a combination of REST2 enhanced sampling computations and brute force MD simulations.

\section{METHODS}

\section{A. X-ray structures}

The single-crystal X-ray data (Fig. 1a ) reveal a bent molecular geometry of chiral compound S-HC8. Accordingly, columnar layers of S-HC8, orthogonally $\mathrm{H}$-bonded via planar urea-urea ribbons $\left(d_{N H \cdots O=C}=1.9 \AA\right)$, are generated in the solid state. Terminal imidazoles are positioned at one end interacting with two water molecules, while interactions between the alkyl chains stabilize the layers on the other side of each ribbon (Fig. 1e). Analysis of the crystal structure reveals $\mathrm{C}-\mathrm{H} \cdots \mathrm{N}$ interactions $\left(d_{N \cdots H C}=2.7-2.8 \AA\right)$ between two imidazoles, resulting in the formation of orthogonal dimers (Fig. 1a), while the waters are simultaneously H-bonded to both imidazole $\mathrm{N}-\mathrm{H}\left(d_{O \cdots H}=1.9 \AA\right)$ and the vicinal waters $\left(d_{O \cdots H}=1.8 \AA\right)$. The relative asymmetric disposition of the dimers in S-HC8, determines a unique dipolar orientation of the central water molecules, comprising a crystallographic $100 \%$ occupancy of both water H-atoms. The overall structure of chiral S-HC8 shows a unique dipolar orientation for all water-filled S-HC8 I-quartet channels (Fig. 1f). The I-quartet S-HC8 water channels of rhomboidal shape $\left(22.4 \times 14.9 \AA^{2}\right)$ determine a pore-gap in the channel of $2.6 \AA$, enabling the formation of unique dipolar oriented water channels. We assemble blocks of crystal patches into a aggregates to probe the influence of overall size.

\section{B. Systems set up}

In order to study the scaling effect of the membraneembedded imidazole-patch on the water conductance, we performed a series of MD simulations for a range of geometries and sizes of the water channel aggregates.

The packing of the initial patch was based on X-ray singlecristal structures and the composition of lipids follows the one used experimentally ${ }^{10}$, i.e. a lipid mixture with a respective molar ratio 5:4:1 for Cholesterol:POPC:POPS. The ensemble of simulations reported corresponds to variations of the number of S-HC8 molecules in a pre-formed crystal phase. Two design strategies have been adopted to extend the size of an initial patch of these objects. The first strategy is to extend the number of pre-formed adjacent channels along its longest dimension in the membrane plane, referred to as the $\mathrm{x}$-direction (see Fig. 1), from $5 \mathrm{~nm}$ to $13 \mathrm{~nm}$. Alternatively, several blocks, also referred to as rows in the text, have been designed resulting in an extension of the system within the membrane plane along the perpendicular lateral $y$-direction from $3 \mathrm{~nm}$ to $9 \mathrm{~nm}$. In the latter case, the aggregation between different rows is guaranteed by the direction of hydrophobic tails in the membrane plane. The systems are designated as follows, $a x b c h$, where $a$ is the number of rows forming the patch and $b$ is the number of channels per row. If $a$ is equal to $1, a \mathrm{x}$ is omitted.

\section{Molecular dynamics}

All molecular dynamics simulations were performed under periodic boundary conditions with constant pressure. We have restricted ourselves to the use of the CHARMM-36 force field ${ }^{12}$ for lipid molecules, TIP3 $\mathrm{P}^{13}$ for water and the CHARMM General Force Field ${ }^{14}$ (CgenFF) for S-HC8 molecules. Topologies and parameters for all components and assembling of the whole system was obtained using the CHARMM-GUI web interface and readers tools ${ }^{15,16}$. During the solvation step, a concentration of $0.15 \mathrm{M} \mathrm{Na}^{+} \mathrm{Cl}^{-}$ have been added to the solvent, as well as an excess of $\mathrm{Na}^{+}$ ions to neutralize the system charge induced by the negatively charged POPS lipids.

The GROMACS 2018.7 and 2019.2 softwares were used to run the simulations with all atoms, with an integration time step of 2 fs. Bonds involving hydrogen atoms were constrained using the LINear Constraint Solver algorithm ${ }^{17}$. Particle mesh Ewald electrostatics was used with a $12 \AA$ cutoff with the Verlet buffer scheme for nonbonded interactions; the neighbor list was updated every 20 steps. Three baths (S-HC8, lipids, and water and ions) were coupled to a temperature of $310 \mathrm{~K}$ using the Nosé-Hoover thermostat ${ }^{18}$ with a time constant $\tau=1 \mathrm{ps}$ and a chain lengh of 4 . Pressure in the $\mathrm{x} / \mathrm{y}$ dimensions was scaled isotropically with a Parrinello-Rahman barostat $^{19}$ at 1 bar, and the $\mathrm{z}$ dimension was coupled independently to a reference pressure of $1 \mathrm{bar}, \tau=5.0 \mathrm{ps}$, and compressibility of $4.5 \times 10^{-5} \mathrm{bar}^{-1}$. All systems were minimized for 5000 steps with the steepest descent algorithm and equilibrated for $2 \mathrm{~ns}$, using decreasing position restraints of 1000 , 400, 200, 100, $40 \mathrm{~kJ} \mathrm{~mol}^{-1} \mathrm{~nm}^{-2}$ on heavy atoms, with the crystal structure as a reference. Production runs were finally computed without any position restraints during $2000 \mathrm{~ns}$. The choice to extend simulation time up to 3000 ns for larger systems (see Table S1) was motivated by the slow convergence of root-mean-square deviation (RMSD) and permeation rate (see SI Fig. S1 and S2).

\section{Enhanced sampling of conformational sub-states}

Enhanced sampling simulations using REST2 (solute tempering ${ }^{20,21}$ ) methods have been used to improve the quality of the sampling. This method has only been applied to the $12 \mathrm{ch}$ simulation. Indeed, subtle changes of the RMSD of 
central channels observed in prior simulations suggested that conformational transitions may arise in such a large system (see SI Fig. S2), possibly leading to channel and conductance sub-states. We used 12 replicas and scaled the Hamiltonian terms for S-HC8 molecules resulting in an effective temperature ranging from $310 \mathrm{~K}$ to $400 \mathrm{~K}$. The exchange frequency between replicas was set to 4 ps. Exchange probabilities are around $15 \%$ between almost all adjacent replicas, except for the last four replicas where exchange probabilities drop to 0 due to the dislocation of the whole patch.

\section{E. Analysis}

Locally written python scripts using the MDAnalysis ${ }^{22}$ library were used to count the permeation events and assess structural features of the patch deformation. Local Tcl scripts and the Hbonds plugin from the $\mathrm{VMD}^{23}$ software were used to compute RMSD values and mean number of hydrogen bonds (HB). The images were produced with VMD. RMSD calculations are relative to the Xray structure coordinates used as reference, unless otherwise stated. Radius profiles were computed using $\mathrm{Hole}^{24}$ with AMBER united atom radii ${ }^{25}$. A trajectory clustering analysis based on the gromos method ${ }^{26}$ was performed with the Gromacs toolbox on the set of configurations sampled by the REST2 simulations. In the clustering method, RMSD is computed with respect to the first frame of the REST2 simulation. This reference structure corresponds to the last frame of the $12 \mathrm{ch}$ simulation.

To locate permeation events respective to the S-HC8 channels, we tracked each water molecule's trajectory, computing its distance to the channel center of mass (COM) in the membrane plane. The time of a permeation event is defined by the interval of the water molecule crossing the boundary of the channel region delimited by the average position of phosphorus atoms from lipids along the membrane-normal z-axis. In central channels, the channel profile is spatially fluctuating between wide and narrow pores along the z-axis. Therefore the mean pore radius was obtained by averaging the radius profile over $60 \%$ of the central part of the total height of the channel. Variance of the radius along the $\mathrm{z}$-axis provides an additional clue about the local size of the pores lining the channel. To be able to accurately distinguish channel conformations, all radius data was averaged over an evenly weighted window of $10 \mathrm{~ns}$. For the cross analysis of mean pore radius, mean number of hydrogen bonds and permeation rate, we considered the average permeation rate of each channel using a Hanning time window of $200 \mathrm{~ns}$ applied before the permeation time. We verified that changing the position and duration of the time window average has no significant effect on the results. This particular choice improves the distinction between active and inactive channels. For hydrogen bond calculations, we aligned the system using the imidazole headgroup coordinates lining the channel of interest and we considered the number of water molecules in a cylinder of height $28 \AA$ and radius $5 \AA$ centered in the channel center of mass (COM). Because of the high stability of central channels, this approach guarantees that no water molecules from the upper and lower mouth of the chan- nel nor from adjacent channels are counted. We counted the hydrogen bond interactions between water molecules themselves $\left(\mathrm{H}_{2} \mathrm{O} \leftrightarrow \mathrm{H}_{2} \mathrm{O}\right)$, between water and amine nitrogen acceptors from imidazoles lining the channel $\left(\mathrm{H}_{2} \mathrm{O} \leftarrow\right.$ Imid $)$, between water and protonated nitrogen donors from imidazoles lining the channel $\left(\mathrm{H}_{2} \mathrm{O} \rightarrow\right.$ Imid $)$ and between water and lipid molecules $\left(\mathrm{H}_{2} \mathrm{O} \leftrightarrow\right.$ lip $)$.

\section{RESULTS}

\section{A. Effect of size and geometry of the channel patches on water conductance}

In this first section we quantify the average water conductance in the simulated artificial channel systems normalised with respect to the number of effective channels, including the peripheral pores at the edge in direct contact with the lipid membrane. The water permeation dynamics is reported in Fig. 2 for each system as a function of time. After a transient fast increase, the permeation reaches a steady regime well described by a linear behaviour. It is worth noting that for all but one of the systems, the steady behaviour is reached after 700 ns (see SI Fig. S1.c and d), while the largest one $(2 \times 12 \mathrm{ch})$ shows a clear change of conductance after $2 \mu \mathrm{s}$. The time evolution of the conductance regime is characterized by block analysis and reported in SI Fig. S1.d.

It is important to point out that the simulations start from ad hoc designed patches built from a crystal template and inserted in the membrane. Both lipid rearrangement and SHC8 molecule reorientation (see SI Fig. S1-S2) take place in the transient phase. Once the structure is stabilised, an average conductance per channel can be extracted from the slope measured in the linear regime, see Table I, that is within the range of $15-23 \times 10^{6} \mathrm{H}_{2} \mathrm{O} / \mathrm{s} /$ channel. It should be noted that the actual calculated conductance rate overestimates over one order of magnitude the experimentally observed value. One reason for this discrepancy may be the relative importance of lateral over central channels because of the relatively small system size simulated. Patches in the experimental systems for which no size measurements currently exist - may well be significantly larger, with a major fraction of "central" channels, thereby reducing the discrepancy between computationally predicted and experimentally measured permeation rates. Furthermore, we previously underlined the strong dependence of the quantitative measurements of such simulations on the force field and the water model ${ }^{27}$. Note that the conductance of lateral channels is consistent with previous studies, with small differences due to the lack of statistics at this time ${ }^{11}$ (see SI Table S2). For central channels, discrepancies with previous studies arise, as we detected a new pattern of increased conduction we were not observing in smaller systems. Visual inspection suggests that new conformational sub-states may be responsible for the higher permeation regime of the central channels. We will investigate this conformational parameter separately from the crystal patch size parameter influence. It should however be noted that these putative sub-states were only observed for the larger aggregates, which might condi- 


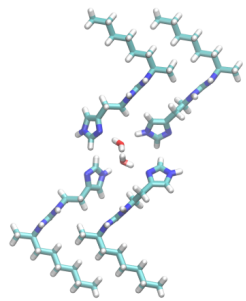

(a)

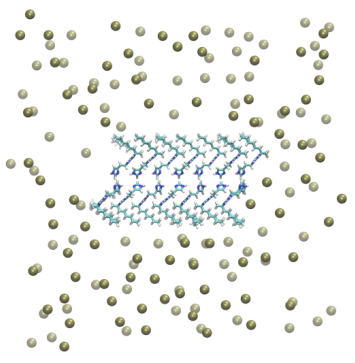

(b)

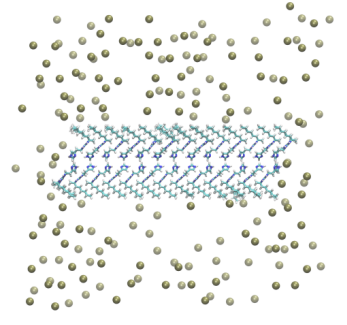

(c)

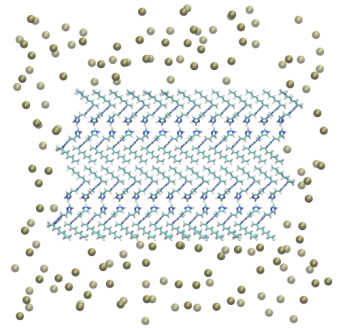

(d)
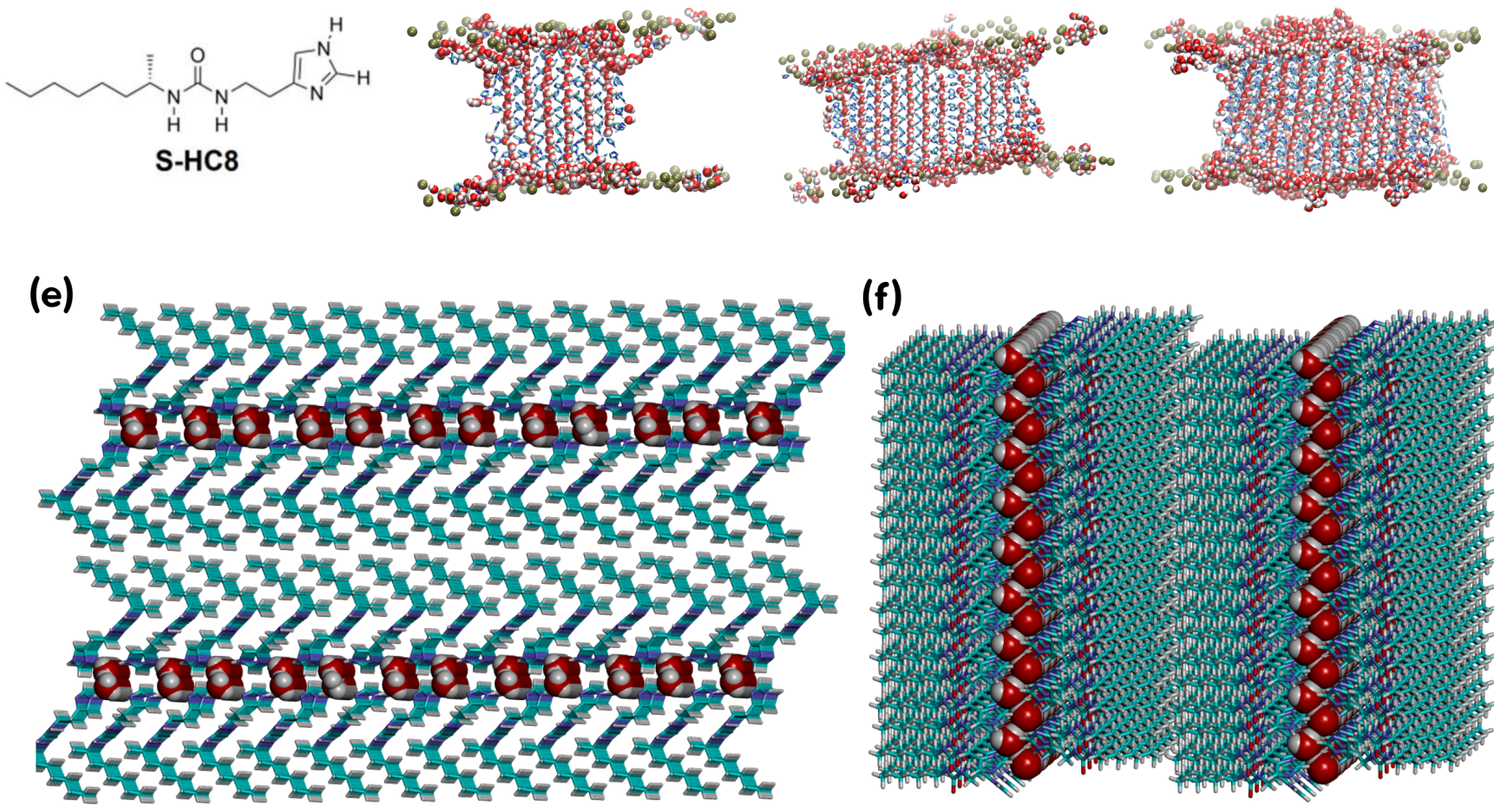

(f)

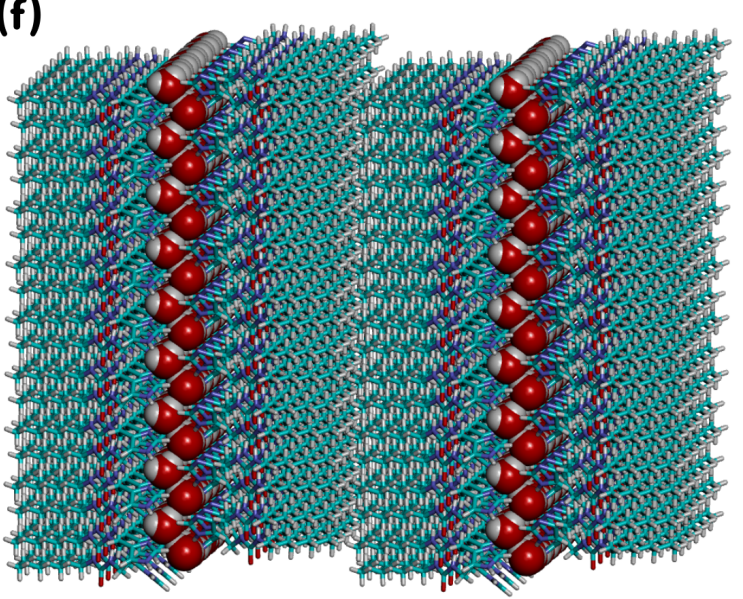

FIG. 1. Initial aggregation setups for molecular dynamics simulations.(a) Top view in stick representation (N, blue, C, cyan, O, red, H-white) of the water confined I-quartet X-ray single crystal structures of S-HC8; (b),(c),(d) views of the initial frame (top) and lateral view of the final frame (bottom) of molecular dynamics simulations built with respectively 6,12 and 2x12 preformed channels. Only the molecules of the S-HC8 compound and the phosphorus atoms of the lipids are represented in the upper panels; water molecules close to S-HC8 compounds, imidazole polar heads and the phosphorus atoms of the lipid headgroups are shown in the bottom panels. Hydrophobic tails of the S-HC8 compound and all lipids are omitted in these pictures for clarity; (e) Top and (f) lateral view crystal packing chiral non-centrosymmetric S-HC8 confining oriented water-wires with a unique dipolar orientation for total water-filled I-quartet channels.

tion the occurrence of these states.

As we reported in previous studies ${ }^{11,27}$, permeation events mainly occur through lateral and exposed channels in contact with lipids. Nevertheless, some distinct patterns of central permeation appear in the larger systems. All central channels found in the crystal structures remain hydrated during the whole simulation time but only a few ones stay in an active conducting regime characterised by a permeability comparable to that of the lateral channels. In some cases, central channels undergo a series of transitions from an active to an inactive conformation with a broad distribution of conducting times between $100 \mathrm{~ns}$ and $1800 \mathrm{~ns}$ (see SI Fig. S3). Furthermore the overall permeation of the central and lateral channels reaches the steady regime at different times (see SI Fig. S1).
These results indicate that the channel assisted water transport across the membrane involves multiple timescales, each associated to different molecular relaxation and stabilization mechanisms.

\section{B. Patterns of permeation}

We now turn our attention more specifically to the pattern of water transport. In Fig. 3 we report the water permeation for individual channels and its spatial localisation with respect to the membrane surface for the representative systems $6 \mathrm{ch}$, $12 \mathrm{ch}$, and $2 \times 12 \mathrm{ch}$.

The permeation along the channels at the edge of the patch 
TABLE I. Water conductance values along one direction reported for the set of plain MD simulations. Results from previous experimental measurements are provided for comparison. Water conductance values are computed using number of permeation events in a time window normalized by the number of effective channels in the preformed crystal patch and divided by a factor of 2 to account for unidirectional flow).

\begin{tabular}{ccc}
\hline \hline Simulation & Nb. of effective channels & $\begin{array}{c}\text { Conductance } \\
\left(\times 10^{6} H_{2} \mathrm{O} / \text { s/channel }\right)\end{array}$ \\
$6 \mathrm{ch}$ & 8 & 19 \\
$2 \times 6 \mathrm{ch}$ & 14 & 24 \\
$3 \times 6 \mathrm{ch}$ & 24 & 19 \\
$12 \mathrm{ch}$ & 14 & 14 \\
$2 \times 12 \mathrm{ch}$ & 28 & $15^{\mathrm{a}}$ \\
$\exp ^{5}$ & - & 1.4
\end{tabular}

a conductance computed in the interval $[2 \mu \mathrm{s}, 3 \mu \mathrm{s}]$; during equilibration interval [1-2 $\mu$ s], the conductance is higher : $31.10^{6} \mathrm{H}_{2} \mathrm{O} / \mathrm{s} /$ channel

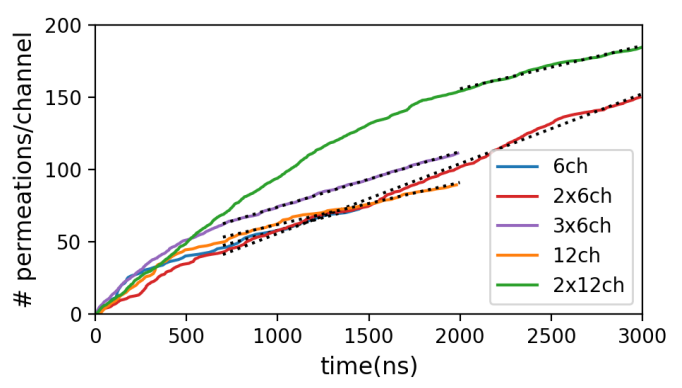

FIG. 2. Time series of number of permeation events normalized over the total number of pre-formed S-HC8 channels in the membrane. Achieving a constant permeation rate after an initial time offset provides a simple measure that the system reaches a converged transport state. Water conductance values associated to each simulation are reported in Table 1.

involves a broad spread in the locations in the $x-y$ plane. This variability results from the higher mobility and relative rotation of the external molecules forming the patch within the upper and lower membrane leaflets.

Secondly, it is worth noting that the flux of water is balanced along both directions, confirming that the diffusion process is operating under equilibrium. While the low level of permeation in the central channels for the small system $6 \mathrm{ch}$ has already been reported ${ }^{11}$, we present here for the first time the emergence of conduction patterns in the central core of the larger assemblies. For the systems $12 \mathrm{ch}$ and $2 \times 12 \mathrm{ch}$, the water transport is localised at specific locations, presenting an almost regular alternation among individual or a pair of conducting channels with non-conducting ones. The patterns are well represented by the water transport magnitude shown in the bottom panels of Fig. 3. The level of water conduction reported in the central part of the large $12 \mathrm{ch}$ and $2 \mathrm{x} 12 \mathrm{ch}$ systems matches that quantified in-silico for another artificial channelforming molecule studied in the past, $\mathrm{R}-\mathrm{HC} 8^{11}$.

\section{Enhanced sampling for central channel activation}

We observed a long relaxation time for the $12 \mathrm{ch}$ setup system until the steady regime for conductance was attained. To address this issue, we resorted to enhanced sampling simulations. This strategy allowed us to explore how the average conduction and the emerging pattern for central channel activation depend on the initial configuration of the patch, independently from the block size effect. For this purpose we performed REST2 simulations as detailed in the Methods section. From the generated trajectories at effective temperatures ranging from $310 \mathrm{~K}$ up to $348 \mathrm{~K}$ we quantified the deformation of the patch by computing the root mean square displacement of the atoms forming the central channel (index 7) with respect to the initial configuration. The resulting distribution is plotted in Fig. 4.a. For the lowest effective temperature, the RMSD distribution shows two main peaks at around 0.5 and $1 \AA$ with a third state appearing at $1.5 \AA$. Raising the temperature enables sampling of more distorted configurations as visible by the increased populations of states at higher RMSD values. By performing a clustering procedure based on the RMSD metric, we extracted three representative states of the patch's conformational landscape (star symbols in Fig. 4.a). Starting new simulations for each of these configurations, we performed brute force MD and quantified individual channel conductance. The final results are reported in Fig. 4.b showing that different water transport patterns exist depending on the initial conformational state. In system $12 \mathrm{ch}^{a}$ similarly to what was observed starting from the $\mathrm{x}$-ray configuration both central channels allow for permeation, while for systems $\operatorname{ch} 12^{b, c}$ only one of the central channels is activated for water conduction that can be as high as that measured in lateral channels (see $12 \mathrm{ch}^{b}$ ). The overall average per channel conductance computed over the whole system varies between 5.8 and $12 \times 10^{6} \mathrm{H}_{2} \mathrm{O} / \mathrm{s} /$ channel, see SI Table S2. Finally, we observe that the activation of the water transport depends on the size of the channel. This is evident by following in parallel the time evolution of the pore radius and of water transport. This correlation is presented in Fig. 4.c for the $12 \mathrm{ch}^{a, b, c}$ systems. The plotted timelines seem to indicate the existence of a threshold. For pore sizes larger than $1.4 \AA$ the channel is activated for water transport, while for smaller pore radii it is not. This correlation will be discussed in detail in the next section.

\section{Correlation among permeation event, pore size and hydrogen bond connectivity}

In this final section we explore the correlation among permeation events, water molecular packing and hydrogen bond connectivity inside the pores. We use the whole set of simulations to gather an extended body of statistical data. The results of our investigation are shown in Fig. 5. In panel a of the figure, each permeation event is associated to the average pore radius experienced by the diffusing water and shown in a scatter plot where the colour of each event indicates the pore radius variance estimated during the time window of the permeation. The data reveal the existence of three distinct per- 


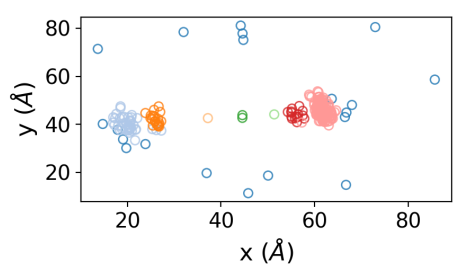

(a)

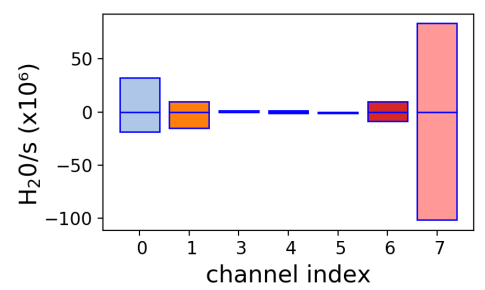

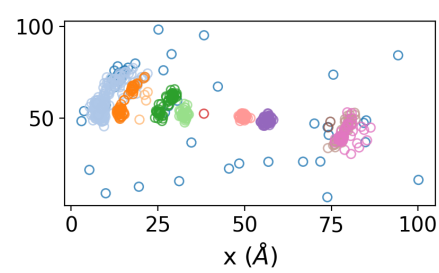

(b)

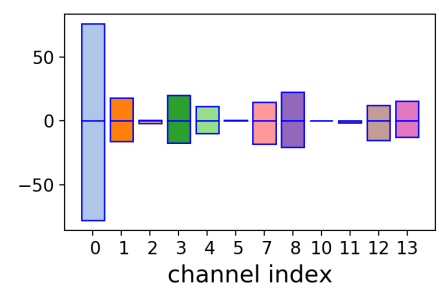

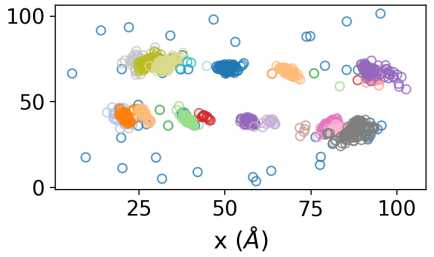

(c)

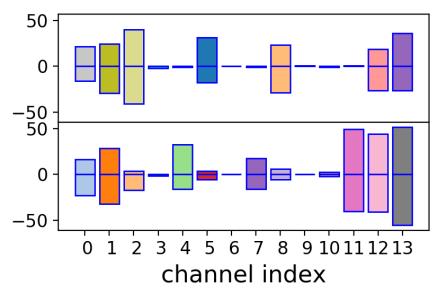

FIG. 3. Water permeation event distribution normalized over the time window on which total water conductance is considered as constant in time, see Fig.2 . Permeation event distribution in the plane of the membrane (top panels) and water conductance per channel (lower panels; looking down on the membrane plane with channels horizontally aligned) for systems $6 \mathrm{ch}(\mathrm{a}), 12 \mathrm{ch}(\mathrm{b})$ and $2 \times 12 \mathrm{ch}(\mathrm{c})$. Data collection has been initiated after the same threshold as used for the calculation of the total conductance, see Fig.1. Positive and negative values account for permeation events that take place in both directions across the membrane. Figuratively speaking this would correspond to water molecules coming out of or going into this sheet of paper in the top row pictorial representation. Channel indices follow the spatial arrangement from left to right; the channels at the ends of each row are effective channels delimited by both lipid and S-HC8 molecules (e.g. channel ID 0 and 7 for simulation $6 \mathrm{ch}$ ). A unique color is associated to each channel ID, for consistent labeling and identification of the channel in both upper and lower panels.

meation regimes.

A low permeation regime accounts for rare translocation events through narrow channels with water molecules mainly organised in a single file wire. In these structures, as well as in non-conducting channels, each water molecule forms on average $3.46 \pm 0.24$ hydrogen bonds with the imidazole groups and with other waters $\left(\mathrm{H}_{2} \mathrm{O} \leftrightarrow \mathrm{H}_{2} \mathrm{O}: 1.62 \pm 0.18\right.$; $\mathrm{H}_{2} \mathrm{O} \rightarrow$ Imid : $0.93 \pm 0.09 ; \mathrm{H}_{2} \mathrm{O} \leftarrow$ Imid : $0.90 \pm 0.10$ ), see SI Fig. S5, thus experiencing a connectivity similar to a water molecule in bulk solution. In our previous study ${ }^{11}$, we already identified such slow processes which can be due to rare permutations between two molecules in an almost perfect single-file conformation. The statistics of these rare events however remain very poor and insufficient to quantify precisely the single-channel permeability on this regime.

The medium and high permeation regimes share similar features. They occur when the pore radius exceeds a threshold value of ca. $1.34 \AA$. The high conductance regime is associated to larger pore sizes, with the pore radius averaging around $1.45 \AA$ and experiencing larger fluctuations. The large fluctuations of the pore radius along the channel axis are accompanied by the local breaking of the water single-file wire. The associate fluctuations of the water dipolar orientation enable some water molecules to create exclusive contacts with the channel molecules at the expense of the contact with water molecules, leading to an increase in the number of water molecules in the channel, with decreasing HB connectivity, and therefore resulting in single channel permeability on the order of $30 \times 10^{6} \mathrm{H}_{2} \mathrm{O} / \mathrm{s} /$ channel.

In this regime the diffusing water molecules experience the weakest $\mathrm{HB}$ connectivity, with the average number of formed
$\mathrm{HB}$ of $3.07 \pm 0.10\left(\mathrm{H}_{2} \mathrm{O} \leftrightarrow \mathrm{H}_{2} \mathrm{O}: 1.33 \pm 0.06 ; \mathrm{H}_{2} \mathrm{O} \rightarrow\right.$ Imid : $1.04 \pm 0.04 ; \mathrm{H}_{2} \mathrm{O} \rightarrow$ Imid : $0.70 \pm 0.08$ ).

By visual inspection, we saw that high permeation rates were triggered by a quick reorientation of both concerted water molecules in the channel and imidazole hydrophilic heads. Furthermore the transition from "medium" to "high" state of permeation arises when one of the two channels stops conducting while the other one becomes highly permeable (see SI Fig. S6). This observation suggests a strong coupling between the transport activity of two adjacent channels (in a given row), the larger dimension of one pore that allows for high permeability constrains the dimension of the neighbouring one forcing it to lower transport activity. We did not witness a similar effect in the perpendicular direction between adjacent rows. A detailed molecular representation of the permeation process is reported in SI Fig. S7.

\section{CONCLUSION}

During the last two decades water structure in confined environments has been extensively studied in the framework of nanotubes $^{28,29}$, hydrophobic pores ${ }^{30}$ and artificial hydrophobic channels ${ }^{2}$. The low friction in hydrophobic pores results in a high water permeability, comparable to Aquaporin channels found in nature $\left(10^{9} \mathrm{H}_{2} \mathrm{O} / \mathrm{s} /\right.$ channel $)$. To our knowledge, no significant improvement has been achieved in numerical studies of hydrophilic pores. In such confined hydrophilic environments, water structure is ordered in singlefile wires and high friction due to hydrogen bond connectivity with molecules of the pore walls results in a much smaller 


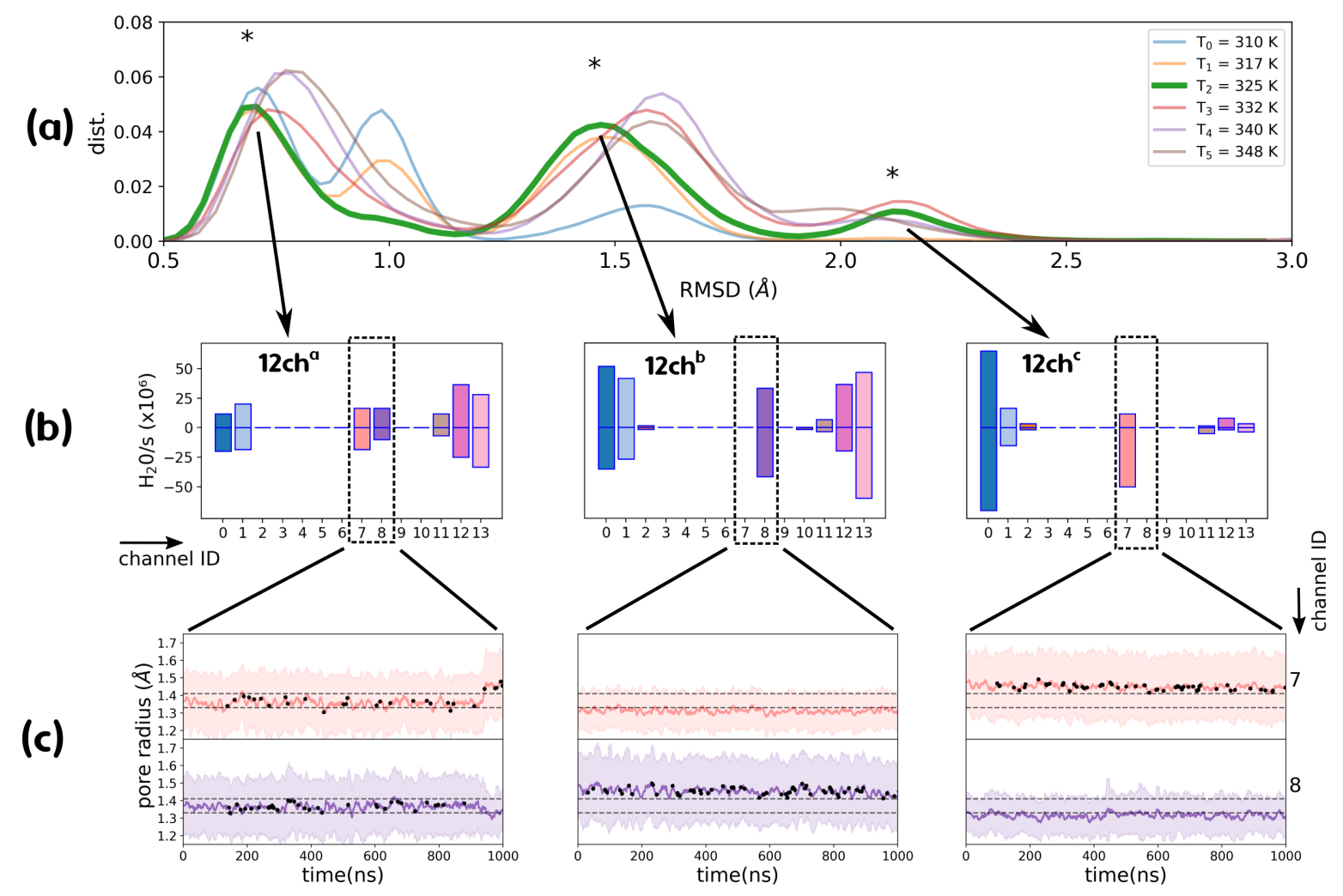

FIG. 4. a) RMSD distribution of the imidazole headgroup structure of channel ID 7 in the 12ch simulation during a 90ns-long REST2 simulation. Each line accounts for a different replica with lowered energy barriers or equivalent high temperature for atoms belonging to the channel. The third replica simulation at $T_{2}=325 \mathrm{~K}$ (thick green line) was used as seed for three independent simulations. Starting structures were obtained after RMSD clustering and are indicated by a* symbol; (b) Water conductance per channel in the three MD simulations with initial structures derived from the REST2 approach $(*$ symbol, panel a). Left, middle and right panels correspond to each of the three independent MD simulations $\left({ }^{a},{ }^{b},{ }^{c}\right)$ starting from three initial structures (see SI Fig. S4) with respective RMSD of 0.7, 1.4, 2.1 A (c) Timelines of mean pore radius (pink and mauve lines) and spatial fluctuations of the pore profile (pink and mauve colored backgrounds) in selected central channels (ID 7 and 8) of the three MD simulations mentioned above and shown in panel (b). Superimposed black dots indicate permeation events in the corresponding channel.

water conductance $\left(10^{9} \mathrm{H}_{2} \mathrm{O} / \mathrm{s} /\right.$ channel $)$. Yet, we reported in the actual study a possible enhanced transport of water through subnanometric hydrophilic pores with a conductance of $30 \times 10^{6} \mathrm{H}_{2} \mathrm{O} / \mathrm{s} /$ channel. Using molecular dynamics simulations and an enhanced sampling approach, we were able to characterise several permeation regimes. High conduction regimes are associated to an increase of the pore radius up to $1.45 \AA$. This widening coincides with a local perturbation of single-file wires and a smaller number of hydrogen bonds compared to the bulk or inactive channels. The number of hydrogen bonds that water molecules can form in biological channels such as Aquaporins is a major determinant for the osmotic single-channel permeability for single-water wires ${ }^{9,31}$.

Interestingly, we noticed that the value of per channel water permeation strongly varies along the patch, with very high values generally observed at the extremities. At the extremities the conductance is much higher than the experimentally reported one, whereas it is quite comparable in central channels. For now, we do not know exactly the size of the aggregates inserted in the membrane, but we can expect that for large nanoscopic structures, the dominating contribution to the overall permeability will be given by central regions, as expected by comparing the value of conductance.

Although we restricted ourselves to equilibrium conditions, we observed a similar effect in the case of AWCs based on self-assembled systems in which highly-ordered channels can undergo conformational changes with a favoured state with low number of hydrogen bonds. However these structural changes of water have to be challenged under non-equilibrium conditions such as electrical fields, osmotic and hydrostatic pressure $^{32-34}$. A coarse-grain approach ${ }^{35,36}$ applied to the specific treatment of synthetic materials would be necessary to access significantly longer timescales and to probe scalability for the future applications in inverse osmosis and ultrafiltration devices ${ }^{37,38}$. 

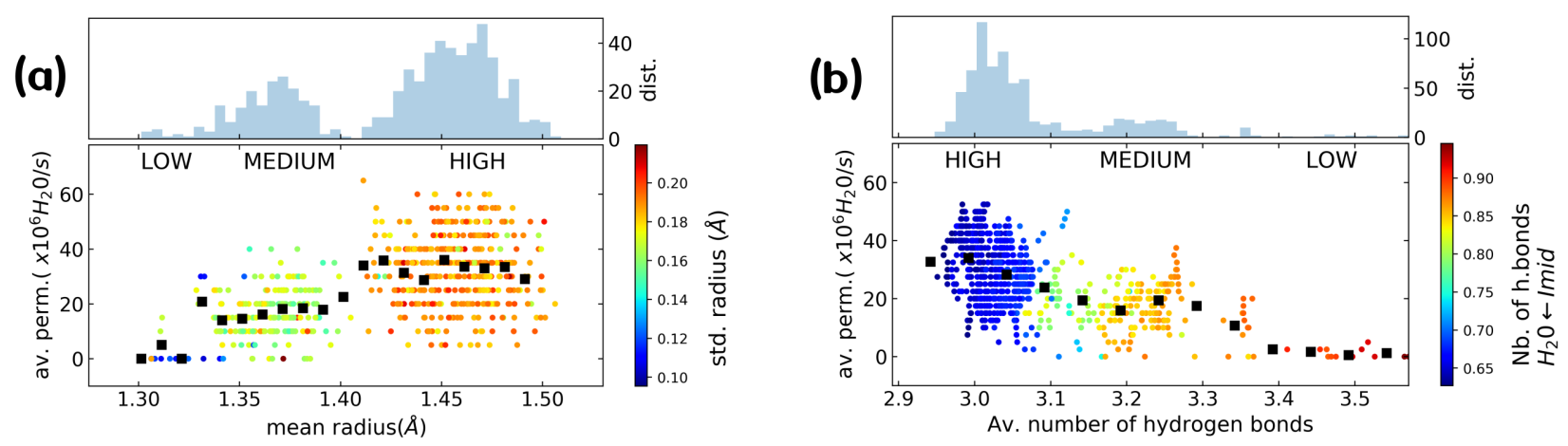

FIG. 5. Averaged water conductance over a constant time window of $200 \mathrm{~ns}$ prior to each permeation event accounted for in central channels with respect to its mean pore radius (a, lower panel) and the mean number of hydrogen bonds of water molecules (b, lower panel) and their corresponding distribution in the dataset (upper panels). Statistical data have been collected over all simulations reported in SI Table S1. The reported observables have been first computed at each nanosecond frame of the simulation and the point values reported in the figure result from a Hanning window averaging during 200ns. In panel a, as the pore profile may vary along the z-axis, the mean pore radius is a spatial mean value. Spatial fluctuations are also encoded in standard deviation of pore radius (colored scale). In panel b), the mean number of hydrogen bonds is computed from all the hydrogen bonds that water can form in a central channel, namely with imidazole donors and acceptors, water molecules and lipids (eventually zero). The coloring map refers to particular cases of imidazole donor-water hydrogen bonds that play a role in the transport activity.

\section{ACKNOWLEDGEMENTS}

This work was supported by Agence Nationale de la Recherche ANR-18-CE06-0004-02, WATERCHANNELS. Computational work was performed using HPC resources from GENCI (grant number A0070701714) and from LBTHPC thanks to support from Geoffrey Letessier. This work was supported by the "Initiative d'Excellence" program from the French State (Grant "DYNAMO", ANR-11-LABX-0011 and grant "CACSICE", ANR-11-EQPX-0008). M.B. thanks Sesame Ile-de-France for co-funding the display wall used for data analysis.

\section{SUPPORTING INFORMATION}

${ }^{1}$ F. Fornasiero, H. G. Park, J. K. Holt, M. Stadermann, C. P. Grigoropoulos, A. Noy, and O. Bakajin, "Ion exclusion by sub-2-nm carbon nanotube pores," Proceedings of the National Academy of Sciences 105, 1725017255 (2008), publisher: National Academy of Sciences Section: Research Articles.

${ }^{2}$ Y.-x. Shen, W. Si, M. Erbakan, K. Decker, R. D. Zorzi, P. O. Saboe, Y. J. Kang, S. Majd, P. J. Butler, T. Walz, A. Aksimentiev, J.-1. Hou, and M. Kumar, "Highly permeable artificial water channels that can self-assemble into two-dimensional arrays," Proceedings of the National Academy of Sciences 112, 9810-9815 (2015), publisher: National Academy of Sciences Section: Physical Sciences.

${ }^{3}$ X.-B. Hu, Z. Chen, G. Tang, J.-L. Hou, and Z.-T. Li, "Single-Molecular Artificial Transmembrane Water Channels," Journal of the American Chemical Society 134, 8384-8387 (2012), publisher: American Chemical Society. ${ }^{4}$ X. Zhou, G. Liu, K. Yamato, Y. Shen, R. Cheng, X. Wei, W. Bai, Y. Gao, H. Li, Y. Liu, F. Liu, D. M. Czajkowsky, J. Wang, M. J. Dabney, Z. Cai, J. Hu, F. V. Bright, L. He, X. C. Zeng, Z. Shao, and B. Gong, "Self- assembling subnanometer pores with unusual mass-transport properties," Nature Communications 3, 949 (2012), number: 1 Publisher: Nature Publishing Group.

${ }^{5}$ E. Licsandru, I. Kocsis, Y.-x. Shen, S. Murail, Y.-M. Legrand, A. van der Lee, D. Tsai, M. Baaden, M. Kumar, and M. Barboiu, "Salt-Excluding Artificial Water Channels Exhibiting Enhanced Dipolar Water and Proton Translocation," Journal of the American Chemical Society 138, 5403-5409 (2016).

${ }^{6}$ Y. D. Yuan, J. Dong, J. Liu, D. Zhao, H. Wu, W. Zhou, H. X. Gan, Y. W. Tong, J. Jiang, and D. Zhao, "Porous organic cages as synthetic water channels," Nature Communications 11, 4927 (2020), number: 1 Publisher: Nature Publishing Group.

${ }^{7}$ J. Shen, R. Ye, A. Romanies, A. Roy, F. Chen, C. Ren, Z. Liu, and H. Zeng, "Aquafoldmer-Based Aquaporin-like Synthetic Water Channel," Journal of the American Chemical Society 142, 10050-10058 (2020), publisher: American Chemical Society.

${ }^{8}$ L.-B. Huang, M. Di Vincenzo, Y. Li, and M. Barboiu, "Artificial Water Channels: Towards Biomimetic Membranes for Desalination,” Chemistry A European Journal n/a (2020), 10.1002/chem.202003470, publisher: John Wiley \& Sons, Ltd.

${ }^{9}$ A. Horner and P. Pohl, "Single-file transport of water through membrane channels," Faraday Discussions 209, 9-33 (2018), publisher: The Royal Society of Chemistry.

${ }^{10}$ I. Kocsis, M. Sorci, H. Vanselous, S. Murail, S. E. Sanders, E. Licsandru, Y.-M. Legrand, A. v. d. Lee, M. Baaden, P. B. Petersen, G. Belfort, and M. Barboiu, "Oriented chiral water wires in artificial transmembrane channels," Science Advances 4, eaao5603 (2018), publisher: American Association for the Advancement of Science Section: Research Article.

${ }^{11}$ S. Murail, T. Vasiliu, A. Neamtu, M. Barboiu, F. Sterpone, and M. Baaden, "Water permeation across artificial I-quartet membrane channels: from structure to disorder," Faraday Discussions 209, 125-148 (2018).

${ }^{12}$ J. B. Klauda, R. M. Venable, J. A. Freites, J. W. O'Connor, D. J. Tobias, C. Mondragon-Ramirez, I. Vorobyov, A. D. MacKerell, and R. W. Pastor, "Update of the CHARMM All-Atom Additive Force Field for Lipids: Validation on Six Lipid Types," The Journal of Physical Chemistry B 114, 7830-7843 (2010), publisher: American Chemical Society. 
${ }^{13}$ W. L. Jorgensen, J. Chandrasekhar, J. D. Madura, R. W. Impey, and M. L. Klein, "Comparison of simple potential functions for simulating liquid water," The Journal of Chemical Physics 79, 926-935 (1983), publisher: American Institute of Physics.

${ }^{14} \mathrm{~K}$. Vanommeslaeghe and A. D. MacKerell, "Automation of the CHARMM General Force Field (CGenFF) I: Bond Perception and Atom Typing," Journal of Chemical Information and Modeling 52, 3144-3154 (2012), publisher: American Chemical Society.

${ }^{15} \mathrm{~S}$. Jo, T. Kim, V. G. Iyer, and W. Im, "CHARMM-GUI: A web-based graphical user interface for CHARMM," Journal of Computational Chemistry 29, 1859-1865 (2008), _eprint: https://onlinelibrary.wiley.com/doi/pdf/10.1002/jcc.20945.

${ }^{16}$ S. Kim, J. Lee, S. Jo, C. L. Brooks, H. S. Lee, and W. Im, "CHARMM-GUI ligand reader and modeler for CHARMM force field generation of small molecules," Journal of Computational Chemistry 38, 1879-1886 (2017).

${ }^{17}$ B. Hess, H. Bekker, H. J. C. Berendsen, and J. G. E. M. Fraaije, "LINCS: A linear constraint solver for molecular simulations," Journal of Computational Chemistry 18, 1463-1472 (1997), _eprint: https://onlinelibrary.wiley.com/doi/pdf/10.1002/\%28SICI\%291096987X\%28199709\%2918\%3A12\%3C1463\%3A\%3AAIDJCC4\%3E3.0.CO\%3B2-H.

${ }^{18}$ S. D. Bond, B. J. Leimkuhler, and B. B. Laird, "The Nosé-Poincaré Method for Constant Temperature Molecular Dynamics," Journal of Computational Physics 151, 114-134 (1999).

${ }^{19}$ M. Parrinello and A. Rahman, "Crystal Structure and Pair Potentials: A Molecular-Dynamics Study," Physical Review Letters 45, 1196-1199 (1980), publisher: American Physical Society.

${ }^{20}$ L. Wang, R. A. Friesner, and B. J. Berne, "Replica exchange with solute scaling: a more efficient version of replica exchange with solute tempering (REST2)," The Journal of Physical Chemistry. B 115, 9431-9438 (2011).

${ }^{21}$ G. Stirnemann and F. Sterpone, "Recovering Protein Thermal Stability Using All-Atom Hamiltonian Replica-Exchange Simulations in Explicit Solvent," Journal of Chemical Theory and Computation 11, 5573-5577 (2015), publisher: American Chemical Society.

${ }^{22}$ N. Michaud-Agrawal, E. J. Denning, T. B. Woolf, and O. Beckstein, "MDAnalysis: A toolkit for the analysis of molecular dynamics simulations," Journal of Computational Chemistry 32, 2319-2327 (2011),_eprint: https://onlinelibrary.wiley.com/doi/pdf/10.1002/jcc.21787.

${ }^{23}$ W. Humphrey, A. Dalke, and K. Schulten, "VMD: Visual molecular dynamics," Journal of Molecular Graphics 14, 33-38 (1996).

${ }^{24}$ O. S. Smart, J. G. Neduvelil, X. Wang, B. A. Wallace, and M. S. P. Sansom, "HOLE: A program for the analysis of the pore dimensions of ion channel structural models," Journal of Molecular Graphics 14, 354-360 (1996).

${ }^{25}$ S. J. Weiner, P. A. Kollman, D. A. Case, U. C. Singh, C. Ghio, G. Alagona, S. Profeta, and P. Weiner, "A new force field for molecular mechanical simulation of nucleic acids and proteins," Journal of the American Chemical Society 106, 765-784 (1984), publisher: American Chemical Society.

${ }^{26}$ X. Daura, K. Gademann, B. Jaun, D. Seebach, W. F. v. Gunsteren, and A. E. Mark, "Peptide Folding: When Simulation Meets Experiment,"
Angewandte Chemie International Edition 38, 236-240 (1999), _eprint: https://onlinelibrary.wiley.com/doi/pdf/10.1002/\%28SICI\%291521$3773 \% 2819990115 \% 2938 \% 3 \mathrm{~A} 1 / 2 \% 3 \mathrm{C} 236 \% 3 \mathrm{~A} \% 3 \mathrm{AAID}-$ ANIE236\%3E3.0.CO\%3B2-M.

${ }^{27}$ A. Hardiagon, S. Murail, L. Huang, M. Barboiu, F. Sterpone, and M. Baaden, "Stability and structure of adaptive self-organized supramolecular artificial water channels in lipid bilayers," in New Trends in Macromolecular and Supramolecular Chemistry for Biological Applications (Springer, 2020).

${ }^{28}$ F. Zhu and K. Schulten, "Water and Proton Conduction through Carbon Nanotubes as Models for Biological Channels," Biophysical Journal 85, 236-244 (2003).

${ }^{29}$ A. Alexiadis and S. Kassinos, "Molecular Simulation of Water in Carbon Nanotubes," (2008), archive Location: world Library Catalog: pubs.acs.org Publisher: American Chemical Society.

${ }^{30}$ O. Beckstein, P. C. Biggin, and M. S. P. Sansom, "A Hydrophobic Gating Mechanism for Nanopores," The Journal of Physical Chemistry B 105, 12902-12905 (2001), publisher: American Chemical Society.

${ }^{31}$ A. Horner, F. Zocher, J. Preiner, N. Ollinger, C. Siligan, S. A. Akimov, and P. Pohl, "The mobility of single-file water molecules is governed by the number of H-bonds they may form with channel-lining residues," Science Advances 1, e1400083 (2015), publisher: American Association for the Advancement of Science Section: Research Article.

${ }^{32}$ A. Kalra, S. Garde, and G. Hummer, "Osmotic water transport through carbon nanotube membranes," Proceedings of the National Academy of Sciences of the United States of America 100, 10175-80 (2003).

${ }^{33} \mathrm{H}$. Yoshida, S. Marbach, and L. Bocquet, "Osmotic and diffusio-osmotic flow generation at high solute concentration. II. Molecular dynamics simulations," The Journal of Chemical Physics 146, 194702 (2017), arXiv: 1703.02759.

${ }^{34}$ J. Dzubiella, R. J. Allen, and J.-P. Hansen, "Electric field-controlled water permeation coupled to ion transport through a nanopore," The Journal of Chemical Physics 120, 5001-5004 (2004), publisher: American Institute of Physics.

${ }^{35}$ L. Ci, R. S, and S. Msp, "Water in Nanopores and Biological Channels: A Molecular Simulation Perspective.” Chemical Reviews 120, 10298-10335 (2020).

${ }^{36}$ H. Chan and P. Král, "Nanoparticles Self-Assembly within Lipid Bilayers," ACS Omega 3, 10631-10637 (2018), publisher: American Chemical Society.

${ }^{37}$ M. Kumar, J. E. O. Habel, Y.-x. Shen, W. P. Meier, and T. Walz, "HighDensity Reconstitution of Functional Water Channels into Vesicular and Planar Block Copolymer Membranes," Journal of the American Chemical Society 134, 18631-18637 (2012), publisher: American Chemical Society. ${ }^{38}$ Y.-x. Shen, W. Song, D. R. Barden, T. Ren, C. Lang, H. Feroz, C. B. Henderson, P. O. Saboe, D. Tsai, H. Yan, P. J. Butler, G. C. Bazan, W. A. Phillip, R. J. Hickey, P. S. Cremer, H. Vashisth, and M. Kumar, "Achieving high permeability and enhanced selectivity for Angstrom-scale separations using artificial water channel membranes," Nature Communications 9, 1-11 (2018). 